\title{
Desigualdad y democracia por venir
}

Cesáreo Morales

\section{Introducción}

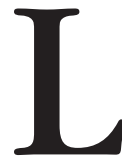

a razón académica, hecha de razones, se acerca o se aleja de la razón pública. Y la razón pública es tomada o no en cuenta por la razón de gobierno. Aquí, en esa perspectiva, se analizan algunos aspectos de la teoría de las decisiones racionales y la teoría de la justicia de Rawls, cuando, sin embargo, la desigualdad aumenta.

\section{Después de la teoría de las decisiones racionales}

Terminada la Segunda Guerra Mundial, la academia estadounidense dio un salto cualitativo en el terreno de la teoría de las decisiones racionales en economía. Su inicio lo marcaron J. von Neumann y O. Morgenstern, con la auténtica revolución teórica propuesta en su libro Theory of Games and Economic Behavior. ${ }^{1}$

Cuando capitalismo y socialismo todavía no definían sus fronteras territoriales y funcionales, la teoría económica avanza construyendo un sujeto presuntamente soberano en sus decisiones que, si bien, se puede equivocar, también soberanamente corrige sus elecciones y preferencias. Un sujeto para el que, aparentemente, todo está a la mano, aun su ignorancia y su información incompleta, superadas por su decisión.

Antes de la decisión, sin embargo, habrá ecuaciones y axiomas que delimitan la ignorancia o la imposibilidad de lo que no se puede conocer, o la imposibilidad lógica o, simplemente, la imposibilidad. Esta soberanía

${ }^{1} \mathrm{~J}$. von Neumann y O. Morgenstern, Theory of Games and Economic Behavior. Princeton, Universidad de Princeton, 1944. 
del individuo sobre su propia ignorancia funda su decisión. Una soberanía que, paradójicamente, en el ejercicio de cierta racionalidad se desintegra a sí misma. Soberanía sin soberanía. Eso son las ecuaciones probabilísticas, formalizaciones de una lotería, aún mejor, de una doble lotería: la de la preferencia, lo que es objeto de la decisión, y la de la decisión misma. Lotería de loterías. Cuando el sujeto cree comprar algo en el mercado se endeuda y entra en un litigio interminable. Y cuando el sujeto se cree libre en su decisión, se pierde. Tan perdido se encuentra y se desencuentra que apenas si es un número cardinal. Un uno más otro uno en el mercado y en otros lugares: ante el recolector de impuestos, frente al policía autoritario, en la fila de votantes en día de elecciones.

Von Neumann y Morgenstern proponen una estructura axiomática matematizada de la decisión llamada racional. La teoría de juegos será la teoría del comportamiento de los individuos en el intercambio económico. Y, según los autores, esta revolución en la economía matemática llevaría, igualmente, a una teoría del poder político y, en general, a una teoría de la "organización social". ${ }^{2}$

Para alcanzar dicho propósito surge una exigencia mayor: identificar los hechos económicos fundamentales para contar con una base empírica segura. Sucede aquí lo mismo que en física, primero han de construirse teorías limitadas a problemas bien circunscritos, para llegar en un momento posterior, a la integración de teorías de mayor alcance. Los complicados mecanismos de los precios, la producción, la ganancia y el gasto de los ingresos, se referirán a un hecho simple en el que encuentran su fundamento: el comportamiento del individuo en el intercambio económico. ${ }^{3}$

Sin embargo, otro hecho desbarata la explicación del comportamiento racional de los individuos en el mercado: la desigualdad. Cuando todos deberían tener ganancias de alguna manera comparables entre ellas, pues todos son racionales, ocurre que el mercado no produce igualdad sino desigualdades. Este efecto no se da aislado, afecta a la economía en su conjunto y, precisamente, la teoría de juegos de Von Neumann y Morgenstern pretende refundar la economía en dos vertientes: las decisiones racionales que aseguren a todos utilidades comparables y la estabilidad de la economía ante la memoria de la crisis económica del 29.

${ }^{2}$ Ibid., pp. 41-43. Vid. Nelson Dresher, Games of Strategy Theory and Applications. Englewood Cliffs, Prentice Hall, 1961; R. B. Braithwate, Theory of Games a Tool for the Moral Philosopher. Cambridge, Mass., Universidad de Cambridge, 1969; Peter Lindert, MACRO a Game of Growth and Policy. Nueva York, Holt, 1970, y Dennis O. Adams, Simulation Games: an Approach to Learning. Worthington, Ch. A. Jones, 1973.

${ }^{3} \mathrm{~J}$. von Neumann y O. Morgenstern, op. cit., p. 8. Vid. Cesáreo Morales, "La matematización en ciencias sociales (¿qué se juega en la teoría de juegos?)”, en Discurso. Cuadernos de Teoría y Análisis, núm. 7, mayo-agosto, 1985, pp. 7-31. 
En términos generales, la economía neoclásica consideró que la estabilización económica se obtenía de manera espontánea y natural en el mercado. ${ }^{4}$ Como prueba de lo anterior, baste con referirse aquí al tratamiento prekeynesiano del equilibrio en el empleo que se obtendría automáticamente a través del juego de la oferta y la demanda.

Keynes echó abajo ese paradigma y propuso el de la intervención del Estado y de los gobiernos: la economía requiere necesariamente de políticas de estabilización. La demanda efectiva es el punto en donde se obtiene un equilibrio estable de la oferta global, del volumen de inversión y de la propensión a consumir. ${ }^{5}$ Desempleo, salarios y "preferencia por la liquidez" exigen políticas que permitan alcanzar el punto de equilibrio y, así, el crecimiento. ${ }^{6}$ La teoría de la estabilidad económica de Keynes fundó el modelo del Estado de Bienestar. Después, H. M. Dobb, expuso el problema de la inestabilidad en una perspectiva marxista. ${ }^{7}$ En 1938, Abraham Bergson diseñó su función de bienestar social a partir de criterios sistemáticos. ${ }^{8}$ Casi diez años más tarde, con una orientación idéntica a la de Bergson, en Foundations of Economic Analysis, ${ }^{9}$ libro que se convirtió en texto clásico, Paul A. Samuelson presentó un modelo más acabado. Con una inteligencia crítica y utilizando elementos de la teoría marxista, G. Myrdal expuso nuevos aspectos del desarrollo económico y la igualdad. ${ }^{10}$

En todo caso, a partir de entonces, dos corrientes teóricas se cruzan, se entremezclan, se oponen entre sí y se complementan. La primera, considera

${ }^{4}$ Vid. Joseph A. Schumpeter, History of Economic Analysis. Oxford, Universidad de Oxford, 1954, pp. 301-311; F. Drucker, "Toward the Next Economics", en Daniel Bell e Irving Kristol, eds., The Crisis in Economic Theory. Nueva York, Harper Colophon Books, 1981, pp. 3-14; Witold Kula, Problemas y métodos de la historia económica. Barcelona, Península, 1977; Mark Blaug, La méthodologie économique. París, Economica, 1982; Michel Didier, Les règles du jeu. París, Economica, 1985, y Paul Vidonne, La formation de la pensée économique. París, Economica, 1986.

${ }^{5}$ John Maynard Keynes, The General Theory of Employment, Interest and Money. Nueva York, Harcourt, 1936, p. 26. Vid. A. Barrière, Controverses sur le système keynésien. París, Economica, 1984.

${ }^{6}$ Vid. Franco Modigliani, "Liquidity Preference and the Theory of Interest and Money”, en Econometrica, 12, 1944, pp. 45-88. Véase también: Gottfried Haberler, Teoría general de Keynes. México, FCE, 1964, y A. W. Phillips, "The Relation between Unemployment and the Rate of Change of Money Wage Rates in the United Kingdom, 1861-1957", en Economica, 25(100), 1958, pp. 283-299.

${ }^{7}$ H. M. Dobb, Political Economy and Capitalism. Londres, Routledge, 1937.

${ }^{8}$ Vid. A. Bergson, "A Reformulation of Certain Aspects of Welfare Economics", en Quarterly Journal of Economics, 52, 1938, pp. 310-334.

9 Paul A. Samuelson, Foundations of Economic Analysis. Cambridge, Mass., Universidad de Harvard, 1947.

${ }^{10} \mathrm{G}$. Myrdal, Political Elements in the Development of Economic Theory. Londres, International Library of Sociology, 1953. 
que el mercado forma parte de un sistema económico cada vez más complejo, la segunda, que una teoría de las elecciones racionales, sean individuales o sociales, habrá de superar, en la práctica, la inestabilidad y los desequilibrios.

Una hunde sus raíces en la economía política clásica y, a querer o no, se acerca a $E l$ capital de Marx, lo que le permite aceptar funcionamientos estabilizadores que después de un punto se vuelven desestabilizadores. ${ }^{11}$ La otra afina las ecuaciones relativas a las decisiones racionales que en su conjunto forman un ente supraindividual. ${ }^{12}$ Ante los cambios en la economía, sobre todo por su internacionalización, después de Keynes, la primera encontró pronto la regulación a través de las distintas formas de la masa monetaria. ${ }^{13}$ La segunda introdujo variaciones en la teoría de las decisiones, al postular la hipótesis de las expectativas racionales. ${ }^{14} \mathrm{Y}$ en una vuelta de tuerca sorpresiva, se observa que la oferta juega en la economía un papel más fundamental que el de simple reacción a la demanda. ${ }^{15}$

En ese horizonte y en los terrenos de un estricto paradigma liberal, preguntándose acerca de las funciones de utilidad de un conjunto social, Kenneth Arrow se enfrentó al problema de dar con una fórmula legítima racionalmente y democrática de agregación de preferencias separadas en un ordenamiento colectivo. Con un rigor sorprendente demostró que, partiendo de las preferencias y decisiones racionales de los individuos, ese ordenamiento del conjunto social es imposible. ${ }^{16}$

${ }^{11}$ Sucedió con la curva de Phillips sobre la relación entre empleo e inflación, una vez que se estabilizó la variación de los salarios. Vid. A. W. Phillips, "The Relation between Unemployment...", en op. cit., pp. 283-299. Igualmente: Richard G. Lipsey, "The Relation between Unemployment and the Rate of Change of the Money Wage Rate in the United Kingdom: 1862-1957: A Further Analysis”, en Economica, 27(105), 1960, pp. 1-31.

12 Vid. C. B. Macpherson, La democracia liberal y su época. Madrid, Alianza, 1981 y C. B. Macpherson, La teoría política del individualismo posesivo. Madrid, Cátedra, 1978. Igualmente: John Dunn, The Political Thought of John Locke. Cambridge, Universidad de Cambridge, 1969; J. Dunn, Rethinking Modern Political Theory. Cambridge, Universidad de Cambridge, 1985, y John Gray, Liberalism. Minneapolis, Universidad de Minnesota, 1986.

${ }^{13}$ Vid. Milton Friedman, "The Role of Monetary Policy", en The American Economic Review, 58, 1968, pp. 1-17; M. Friedman, "The Demand for Money: Some Theoretical and Empirical Results", en The Optimum Quantity of Money and Other Essays. Chicago, Universidad de Chicago, 1969, y M. Friedman, La teoría cuantitativa del dinero. México, FMI, 1975.

${ }^{14}$ Vid. J. F. Muth, "Rational Expectations and the Theory of Price Movements", en Econometrica, 29, 1961, pp. 315-335.

${ }^{15}$ Vid. Lawrence Klein, "The Supply Side", en The American Economic Review, 68, marzo, 1979, pp. 1-7.

${ }^{16}$ K. J. Arrow, Social Choice and Individual Values. 2a. ed. Nueva York, Wiley, 1963, pp. 98-100. La primera edición fue publicada en 1951. 
A su descubrimiento lo llamó "teorema de la imposibilidad", que escuetamente se enuncia así: hay ciertas condiciones de racionalidad que no pueden satisfacer ninguna función de bienestar social. Inicialmente fueron cinco y se reducen a cuatro en la segunda edición de Social Choice and Individual Values (1963). Ellas son:

1. El "dominio irrestricto" (condición U). La función de bienestar social ha de incluir todos los perfiles posibles de preferencias individuales, agregándolas en un solo ordenamiento.

2. La "suma de alternativas relevantes" (condición P). Si todos los individuos de una colectividad prefieren $x$ a $y$, entonces, $x$ es socialmente preferible a $y$.

3. La "independencia de alternativas relevantes" (condición I). La preferencia entre dos ordenamientos sociales, $x$ y $y$, sólo dependen de las preferencias individuales en ambos casos.

4. La "ausencia de dictadura" (condición D). Se prohíbe que la preferencia de una persona por $x$ o $y$ resulte en una preferencia social de $x$ o $y .^{17}$

El teorema de la imposibilidad de Arrow afirma que, si hay al menos tres ordenamientos diferentes y el conjunto de individuos es finito, no existe ninguna función de bienestar social que satisfaga las condiciones U, P, I y D. En otras palabras, cualquier agregación de preferencias que pretenda fundarse en criterios generales de justicia o igualdad, desfallece racionalmente en cuanto a su legitimidad. Un atentado fuerte viene de la no transitividad de las preferencias individuales al orden social que, a la inversa, sí lo son en la regla de mayoría de la democracia. Si ésta suma votos de ciudadanos separados, de acuerdo al supuesto liberal, la misma operación no es aplicable a la forma de repartición de la riqueza. Se cuestiona, entonces, la posibilidad misma del diseño de reglas para alcanzar una sociedad justa. Sólo sería aceptable la evaluación que cada individuo hace de su posición respecto a la de los demás y la conclusión a la que puede llegar de que, aun en desventaja, estaría peor en una situación de menor desigualdad impuesta.

En la segunda edición de Social Choice and Individual Values, el teorema de la imposibilidad se define en términos relacionales puros y coincide con su formalización matemática. Amartya Sen considera "exactamente equivalentes" esta definición relacional y la función matemática de la imposibilidad. ${ }^{18}$ De

${ }^{17}$ Idem.

${ }^{18}$ Amartya Sen, Choice, Welfare and Measurement. Cambridge, Mass., MIT, 1982, p. 10, n. 23, y todo el capítulo ocho de este libro. 
hecho, estas conclusiones remiten directamente al callejón sin salida del utilitarismo liberal frente a la desigualdad social. Los más favorecidos no tienen ninguna razón para aceptar decisiones que disminuyan sus ventajas y los señalados por la mala suerte han de estar satisfechos, a tal punto de no exigir nada que dañe a los primeros. Aquí, la condición P de Arrow, o teorema de Pareto, interviene con toda su fuerza al lado de la condición I, la "independencia de alternativas relevantes". ${ }^{19}$

El teorema de la imposibilidad metió al liberalismo en aprietos y el autor estaba consciente de ello. La neutralidad, por ejemplo, considerada fundamental en la teoría de decisiones, estipula que al adoptarlas no se consideren ventajas que favorezcan a algunos en los ordenamientos de preferencias. Con ello, además de excluir cualquier política de igualdad de resultados, se ponen restricciones mayores a una versión dura de la igualdad de oportunidades. Aunque por razones diferentes, las preferencias de los individuos no desembocan en políticas públicas que impulsen la igualdad. Por un camino distinto, Arrow coincide con el Hayek de Law, Legislation and Liberty, para quien la justicia, alentada de manera compulsiva por la vía estatal, se encuentra siempre contaminada por el autoritarismo. ${ }^{20}$

Amartya Sen hará el milagro teórico de convertir el teorema de la imposibilidad de Arrow en teorema de la multiplicidad de las posibilidades. Esto lo lleva a cabo desde sus primeros trabajos, como se ha anotado un poco antes, hasta los más recientes publicados en su libro The Idea of Justice de 2009. ${ }^{21}$ Arrow en persona, en su misma obra pionera, después de su primera versión pesimista del teorema de la imposibilidad, le llamó "teorema de la posibilidad general". ${ }^{22}$ A partir de aquí, y respecto de la teoría de la justicia, Amartya Sen enumera algunas de las aportaciones de la teoría de la elección social racional: análisis comparativo en vez de principios trascendentales; racionalidad plural; capacidad de corrección; cambios y reformas parciales; complejidad; razonabilidad pública. ${ }^{23}$

En este contexto se concilia la libertad de los individuos y el ordenamiento general elegido por las preferencias de la mayoría. Puesto que un ordenamiento universal a partir de las preferencias de todos es imposible, cada individuo ha de cultivar en sí mismo la tolerancia hacia los otros y hacia el ordenamiento general de preferencias existente, a partir de argumentos racionales y democrá-

${ }^{19}$ Vid. A. Sen, Resources, Values and Development. Cambridge, Mass., Universidad de Harvard, 1984, parte IV, sección 14.

${ }^{20}$ F. von Hayek, Law, Legislation and Liberty. Chicago, Universidad de Chicago, 1976 , p. 67.

${ }^{21}$ A. Sen, La idea de la justicia. México, Taurus, 2010.

22 Ibid., p. 122.

${ }^{23}$ Vid. ibid., pp. 135-140. 
ticos. La conclusión de Amartya Sen es alentadora y quizá lleva hasta sus límites la racionalidad de la propia teoría de las decisiones sociales: "Lo que es, formalmente, un mero resultado de imposibilidad puede tener entonces implicaciones para varios tipos de razonamiento público". Y Sen menciona la discusión sobre el fundamento normativo de las preferencias, las exigencias de la libertad y la revisión de las mismas "normas de razonamiento y de conducta". ${ }^{24}$

Se mantiene el teorema de la imposibilidad, "un hito en la historia de los estudios políticos y sociales tanto como de la economía", ${ }^{25}$ pero inseparable de la posibilidad de distintos ordenamientos no estrictamente racionales para el individuo aislado. Esta posibilidad mantiene su imposibilidad. Amartya Sen explica esta aporía en un artículo en el que hace algunas observaciones sobre la teoría del comportamiento del consumidor de Samuelson. ${ }^{26}$ La preferencia del consumidor es lo que el observador percibe y cuantifica. Pero, la preferencia observada, que tanto Samuelson como Sen llaman "el axioma débil de la preferencia revelada", no explica la decisión del consumidor que se da en un entorno por demás complejo. ${ }^{27}$

Por tanto, se han de distinguir preferencia y decisión. El observador se refiere en primer lugar a la preferencia, pero el consumidor enfrenta primero el proceso de su propia decisión. La preferencia se da en el exterior, la decisión involucra las motivaciones de la voluntad individual y las condiciones de la racionalidad. La preferencia, por eso, es lógicamente transitiva y permite establecer un ordenamiento de acuerdo a las preferencias. Esto es lo que sucede en las elecciones, los votos se suman y ordenan las preferencias por un partido o por un candidato. La decisión, por el contrario, se encuentra en el ámbito individual. Y la intencionalidad guarda sus propios secretos. De aquí que todos los estudios sobre la intención del voto sean engañosos o de resultados falsos, pues lo que en realidad analizan son las preferencias.

Al final de cuentas, si bien las preferencias no explican la decisión, son preferencias. ${ }^{28}$ En realidad, el teorema de la imposibilidad de Arrow es una versión de la indiferencia del individuo racional hacia el ordenamiento de preferencias de una mayoría. ${ }^{29} \mathrm{El}$ individuo sostiene otra racionalidad, pero al final de cuentas opta por preferencias. En las elecciones ningún candidato le satisface, pero vota por el que le parece menos malo. Amartya Sen se refiere al

${ }^{24}$ Ibid., p. 141.

${ }^{25}$ Ibid., p. 309.

${ }^{26}$ P. A. Samuelson, "A Note on the Pure Theory of Consumer's Behavior", en Economica, 5, 1938. Apud A. Sen, "Behavior and the Concept of Preference", en Jon Elster, ed., Rational Choice. Nueva York, Universidad de Nueva York, 1986, p. 60.

${ }^{27}$ A. Sen, "Behavior and the Concept of Preference", en op. cit., p. 61.

${ }^{28}$ Ibid., p. 67.

${ }^{29}$ Ibid., p. 68. 
"asno de Buridan". Este escolástico francés del siglo XIV, para explicar la decisión y la preferencia, relata la anécdota de un asno que, ante dos montones de pasto, mantiene la igualdad de preferencias hasta que muere de hambre. Ésa es la "libertad de indiferencia". Pero, como sostienen Arrow y Sen, la preferencia del individuo, a final de cuentas, es una preferencia que decide entre varias opciones. El costo de la preferencia es que la decisión racional se da siempre en un contexto de "racionalidad limitada", concluye J. C. Harsanyi. ${ }^{30}$ Esto mismo afirma Jon Elster, tanto de la acción colectiva como de los códigos éticos de una sociedad. ${ }^{31}$ De nuevo, las preferencias son aleatorias y sus resultados también son aleatorios: la preferencia, cualquiera sea el proceso de intencionalidad que la acompaña, es una tirada de dados en un juego de lotería. ${ }^{32}$

\section{El neocontractualismo de Rawls}

En 1971, $A$ Theory ofJustice ${ }^{33}$ llamó la atención por la solidez de la propuesta de un acuerdo contractual de corte kantiano, más allá del utilitarismo liberal. ${ }^{34}$ Se reabrió con nuevos argumentos y decisiones técnicas aplicables, la antigua discusión acerca de cómo conciliar justicia y libertades. Los interlocutores de Rawls surgieron de los distintos vértices teóricos vigentes en las dos últimas décadas. ${ }^{35}$

Desde su artículo "Justice as Fairness", de $1958,{ }^{36}$ hasta $A$ Theory of Justice, pasando por "Distributive Justice", de $1967,{ }^{37}$ y en las correcciones

${ }^{30}$ John C. Harsanyi, "Advances in Understanding Rational Behavior", en Jon Elster, ed., Rational Choice, p. 85.

${ }^{31}$ J. Elster, El cemento de la sociedad. Barcelona, Gedisa, 1991, p. 64.

${ }^{32}$ Vid. J. Elster, Domar la suerte. Barcelona/Buenos Aires/México, Paidós, 1991, pp. 105 y 161.

${ }^{33}$ John Rawls, $A$ Theory of Justice. Oxford, Universidad de Oxford, 1972 y J. Rawls, Teoría de la justicia. Madrid, FCE, 1977.

34 Vid. Cesáreo Morales, "Una sociedad justa: legitimidad y gobernabilidad", en Examen, 4, núm. 2, 1992, pp. 14-16; "La justicia en las sociedades complejas (1)", en Examen, 6, núm. 65, 1994, pp. 16-19; "La justicia en las sociedades complejas (2)", en Diálogos sobre filosofía política. México, Asociación Filosófica de México/UNAM, 1995, pp. 137-149.

${ }^{35} \mathrm{El}$ posmarxismo de A. Cohen y J. Habermas; los principales representantes del liberalismo ético como R. Nozik, P. Seabright, R. Dworkin, Th. Angel, T. M. Scanlon y J. C. Harsanyi; filósofos del derecho como H. L. A. Hart y M. Sandel; economistas como Amartya Sen; teóricos de las decisiones y la autoorganización como K. Arrow, A. Buchanan y J. P. Dupuy; hasta Paul Ricoeur y Richard Rorty discutieron sus ideas.

${ }^{36}$ J. Rawls, "Justice as Fairness" [1958], en Collected Papers. Cambridge, Mass., Universidad de Harvard, 1999, pp. 47-72.

${ }^{37}$ J. Rawls, "Distributive Justice" [1967], en Collected Papers, pp. 130-153. 
posteriores, expuso los distintos aspectos de un contrato social del que deriva una poderosa concepción de la justicia. ${ }^{38}$ Para hacerla plausible va armando su teoría. La precondición de la "ausencia de envidia", primero y, luego, en "Distributive Justice", el "velo de la ignorancia", ${ }^{39}$ así como la nueva versión del principio de diferencia, que inicia en ese artículo y completa en Teoría de la justicia.

En "Justice as Fairness", los dos principios de la justicia son enunciados de la siguiente forma: "Primero: toda persona participante en una práctica o afectada por ella, tiene un derecho igual a la libertad más amplia compatible con una libertad igual para todos y, segundo, las desigualdades son arbitrarias, a menos que sea razonable esperar que ellas beneficien a todos, bajo la presuposición de que los lugares y oficios a los que están ligadas o de los cuales provienen, están abiertos a todos". Rawls agrega: "Estos principios expresan una justicia como complejo de tres ideas: libertad, igualdad y recompensa por los servicios que contribuyen al bien común". ${ }^{40}$

La sociedad justa rawlsiana superaría los problemas inherentes al utilitarismo, ciertamente, sobre la base de comparaciones interpersonales entre los individuos que participan en el contrato, pero en las cuales no se ponderan utilidades sino efectos sociales posibles a partir de las reglas acordadas. La "neutralidad" de Arrow se convierte en Rawls en un "juego de negociaciones" entre individuos con capacidad moral, es decir, autointeresados y competentes para evaluar con éxito razonable las consecuencias de sus acciones y las de otros. Eso motivaría el acuerdo en torno a los dos principios de la justicia, que en otra versión, en $A$ Theory of Justice, se enuncian de la siguiente forma: "Primero: toda persona ha de tener un igual derecho a la más amplia libertad básica con una libertad similar para los otros. Segundo: las desigualdades sociales y económicas han de estar en un arreglo que, a la vez, $a$ ) se espere razonablemente que contribuyan a las ventajas de todos, y b) que dependan de posiciones y oficios abiertos a todos". ${ }^{41}$

Teoría de la justicia busca solucionar los problemas encontrados hasta entonces, articulando libertades y principio de diferencia, en un modelo de

38 Vid. Rae Douglas, "Maximin Justice and an Alternative Principle of General Advantage", en The American Political Science Review, 69, junio, 1975, p. 630. Una amplia bibliografía en "A Rawls Bibliography", en Social Theory and Practice, 3 (1), primavera, 1974, pp. 123-127.

${ }^{39}$ Peter Laslett y W. G. Runcimann, eds., Philosophy, Politics and Society (third series). Oxford, Basic Blackwell, 1969, pp. 58-82.

${ }^{40}$ J. Rawls, "Justice as Fairness", en Philosophical Review, 67(2), 1958, pp. 164-194. Publicado posteriormente en P. Laslett y W. G. Runcimann, eds., Philosophy, Politics and Society (second series). Oxford, Basic Blackwell, 1962, p. 133. Aquí se cita de Collected Papers, p. 48.

${ }^{41}$ J. Rawls, $A$ Theory of Justice, p. 60. 
tránsito de la igualdad democrática a la igualdad liberal. ${ }^{42}$ Hasta se puede pensar que intenta solucionar el "teorema de la imposibilidad" de Arrow, aunque nunca lo señale explícitamente, pues apenas se refiere a él en varias notas. Esto, pese a que, sobre todo, en la primera y tercera partes de esa obra, declara su propósito de superar el utilitarismo. Lo intenta oponiendo a éste una base moral que, sin renunciar a las comparaciones interpersonales, otorga la prioridad a las libertades, primer principio, seguida de algunos criterios de eficiencia y equidad en el espacio de los "bienes sociales primarios", segundo principio, y añadiendo que esa jerarquía no se debe modificar.

"Una de las ideas más bellas en la historia de la teoría social y política", la llamó Robert Paul Wolff, quien hizo el inventario de las desilusiones. ${ }^{43}$ Rawls no convenció a casi nadie. Los amantes de las fundamentaciones racionales se rasgaron las vestiduras y los teóricos de las decisiones sentenciaron que el "maximin" de Rawls era inutilizable. Rawls habría fracasado en su intento de definir las reglas de una sociedad justa a partir de las decisiones libres de sus integrantes. Amartya Sen agregó que, para considerar los bienes primarios y hacer relevante el principio de diferencia, era necesario renunciar, por lo menos, a una de las tres primeras condiciones del teorema de Arrow, dominio irrestricto, independencia o el principio de Pareto. ${ }^{44}$ En la discusión, sin embargo, teóricos de las decisiones y aun otros neocontractualistas advirtieron sobre los límites del consenso. ${ }^{45}$

Para Rawls, la sociedad justa sería una sociedad estable, casi sin conflicto. La estabilidad tiene, para él, la misma importancia que el derecho. El contrato ha de alcanzar dicho propósito. La enemistad será neutralizada mediante instrumentos que permitan dirimir controversias sobre intereses contrarios. Del individuo al conjunto social se cuenta con racionalidad deliberativa, equilibrio reflexivo, ordenamientos sociales, consenso diferenciado de la democracia y el lugar central de la Constitución.

Este proyecto deja ver con crudeza las contradicciones que circulan entre derecho y desigualdad. Aun la antropología implícita que lo sustenta, apunta a otros ángulos de las tensiones de cualquier sociedad y las que se dan entre gobernantes y gobernados. Aunque kantiano, Rawls no deja de mirar a Aristóteles. No le interesa tanto la definición de animal político, sino lo que llama "principio aristotélico": "dadas iguales las demás cosas, el ser humano goza el ejercicio de la realización de sus capacidades (sus habilidades innatas o

${ }^{42}$ Ibid., cap. II, sección 13, pp. 75-83.

${ }^{43}$ Robert Paul Wolff, Understanding Rawls. A Reconstruction and Critique of A Theory of Justice. New Jersey, Universidad de Princeton, 1977, p. 16.

${ }^{44}$ Vid. A. Sen, "Behavior and the Concept of Preference", en op. cit., p. 70.

45 Douglas Rae, "The Limits of Consensual Decision", en The American Political Science, 69(4), diciembre, 1975, p. 1923. 
adquiridas), y este gozo aumenta más mientras mejor las despliega o es mayor su complejidad". 46

En Political Liberalism,${ }^{47}$ integrado por ocho conferencias, Rawls vuelve a revisar los principales conceptos de su propuesta y de su exposición. Ahora le interesan, en particular, el trasfondo filosófico del liberalismo político y, sobre todo, el constructivismo kantiano, el consenso racional amplio, la prioridad de lo justo, la razón pública, la estabilidad, la estructura básica de la sociedad y las libertades básicas. El liberalismo político se define como autocontención de los ciudadanos y limitación del poder político. Este mantiene su carácter coercitivo, pero sólo en correspondencia con lo determinado por hombres libres e iguales que se asocian. Si la razón es pluralista en cuanto a religión y moral, también lo será en cuanto al consenso amplio en torno a los principios de una sociedad democrática, justa e igualitaria. También pueden aparecer doctrinas comprensivas "no razonables e irracionales, y hasta demenciales, en cuyo caso el problema es contenerlas para que no socaven la unidad y la justicia de la sociedad". ${ }^{48}$

El núcleo duro del liberalismo constitucional es una concepción política de la justicia como paciencia o razonabilidad democrática. ${ }^{49}$ Ésa sería la única respuesta a la pregunta que cada generación se hace en cuanto a la conciliación de los principios de libertad e igualdad. Los principios suscritos llevarían a una sociedad en la cual se garantizan los derechos fundamentales de todos, y se aplican medidas para asegurar, también a todos, la igualdad de oportunidades. La ambigüedad que permanecía en $A$ Theory ofJustice, "es suprimida y la justicia como imparcialidad es presentada desde el principio como una concepción política de la justicia”. ${ }^{50}$

Se confirma el propósito de Rawls: "generalizar y llevar a un más alto nivel de atracción la doctrina tradicional del contrato social". ${ }^{51}$ A este objetivo es inherente alcanzar una posición conceptual superior al utilitarismo. Se trata del liberalismo igualitario, democrático y constitucional, como una alternativa del utilitarista y el conservador. Se espera que los dos principios de la justicia obtengan un asentimiento racional de los contratantes en un régimen democrático.

${ }^{46}$ J. Rawls, $A$ Theory of Justice, p. 426. En la nota a pie de página explica que "el nombre de 'principio aristotélico' me parece apropiado, teniendo en cuenta lo que Aristóteles dice acerca de las relaciones entre felicidad, actividad y gozo, en la Ética a Nicómaco, libro VII, caps.11-14 y libro x, caps. 1-5".

${ }^{47}$ J. Rawls, Political Liberalism. Nueva York, Universidad de Columbia, 1993 ( $L i$ beralismo político. México, FCE/UNAM, 1995).

${ }^{48}$ Ibid., pp. XVI-XVII.

${ }^{49}$ Ibid., p. XV.

${ }^{50}$ Ibid., p. XVII.

${ }^{51}$ Ibid., p. XV. 
Al mismo tiempo, estos dos principios se fundan en intuiciones políticas de la forma deseable de vivir en la democracia, si se han de alcanzar la libertad, la igualdad política y la igualdad de oportunidades. Aquí se concilian el pluralismo de la libertad religiosa, como lo propuso Hegel en Principios de la filosofía del derecho ( $\$ 270)$, y el constitucionalismo liberal, "como descubrimiento de una nueva posibilidad social: la posibilidad de una sociedad razonablemente armoniosa y estable". 52

Al final de cuentas, parecería que Rawls se proclama heredero de la Ilustración. Poner en juego la razón de todos conduce al liberalismo político de las sociedades democráticas. ${ }^{53}$ La capacidad de razonabilidad de la sociedad llevaría a la estructura moral y razonable del Estado democrático moderno. Sociedad y Estado son producidos por la razonabilidad de los ciudadanos libres e iguales. Las libertades no son puramente formales y la igualdad de oportunidades ha de ser comprobable. Los dos principios de justicia son modificados de acuerdo a este criterio. ${ }^{54}$

"Una concepción política liberal de la justicia", 55 tal es la premisa de Rawls. Eso, de acuerdo a la función de la filosofía política: encontrar una base compartida de principios en cuanto a las instituciones que aseguren libertad e igualdad democráticas. Se trata de partir de la cultura pública misma y de las ideas y principios básicos implícitamente compartidos. De ahí la precondición de la justicia como imparcialidad; un "equilibrio reflexivo". 56

Rawls insiste sobre la naturaleza política de la justicia como imparcialidad o la concepción política de la justicia, no moral ni metafísica. A partir de los dos principios fundamentales se articulan varios elementos.

Primero. Una concepción política referida a la estructura básica de la sociedad o de una democracia constitucional moderna, considerada en ella misma, sin atención a sus relaciones con otras sociedades. ${ }^{57}$ Contiene ideas implícitamente contenidas en la cultura política pública de una democracia. Comprende la sociedad como sociedad cooperativa a través del tiempo, por lo menos, de una generación a la siguiente.

Segundo. La idea de posición original. Tiene que llegarse a un punto desde donde se pueda dar una negociación imparcial, justa. Ésa es la idea de la posición original que Rawls llamó "velo de la ignorancia" ${ }^{58} \mathrm{La}$ idea de posición original conduce a la de representación democrática. Rawls se de-

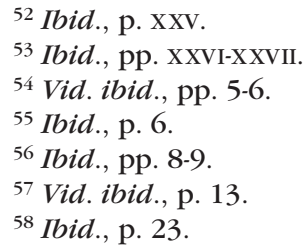


tiene en el pluralismo racional, cuya comprensión es ignorada en la posición original. Lo razonable antes que lo racional da la prioridad a lo justo. "La idea de posición original funciona como medio de reflexión pública y autoclarificación". 59

Tercero. La concepción política de la persona. Fundamentalmente, es la de personas libres. Los ciudadanos se consideran libres unos a otros en la medida en que se ven con "poder moral para tener una concepción del bien". ${ }^{60}$ En una sociedad bien ordenada, los valores políticos y compromisos más generales de ciudadanos libres forman parte de la identidad no institucional o identidad moral. Esos valores son la fuente de auto-autentificación de reclamaciones válidas. Un esclavo no reclama nada. ${ }^{61} \mathrm{El}$ ciudadano libre es libre porque asume responsabilidades.

Cuarto. La sociedad bien ordenada. Demanda tres cosas: todos aceptan los mismos principios de justicia, su estructura básica satisface esos mismos principios y sus ciudadanos poseen un "efectivo sentido de justicia". En esta sociedad, la concepción de justicia públicamente reconocida, establece un punto de vida compartido desde el cual los reclamos de los ciudadanos a la sociedad pueden ser adjudicados". ${ }^{62}$

Quinto. En el punto de partida, cada generación evalúa las condiciones en que se encuentra y elabora la agenda plural de acuerdo a los dos principios de la justicia como imparcialidad. ${ }^{63}$ Se vuelve a validar, entonces, el principio liberal: "el poder político es totalmente legítimo, sólo si es ejercido de acuerdo con una Constitución, cuyos mandatos esenciales es razonable esperar que sean suscritos por todos los ciudadanos en tanto que libres e iguales, a la luz de principios e ideales aceptables a su común razón humana" ${ }^{64}$

Rawls publicó, luego, The Law of Peoples, ${ }^{65}$ honrando así su deuda con Kant, quien, precisamente, termina su Doctrina del derecho con un capítulo que lleva ese título. En una mirada al conjunto de su propia obra, Rawls mantuvo siempre bajo sospecha la "sociedad bien ordenada" y abrigó dudas sobre la "posición original" como presupuesto de la racionalidad de las decisiones de sus miembros. ${ }^{66}$ Al final de Teoría de la justicia ofrece testimonio de ello. "Por tanto, ver nuestro lugar en la sociedad desde la perspectiva de esta posi-

59 Ibid., p. 26.

${ }^{60}$ Ibid., p. 30.

${ }^{61}$ Ibid., p. 33.

${ }^{62}$ Ibid., p. 35.

${ }^{63}$ Ibid., p. 134.

${ }^{64}$ Ibid., p. 137.

${ }^{65} \mathrm{~J}$. Rawls, The Law of Peoples. Cambridge, Mass., Universidad de Cambridge, 1999. (El derecho de gentes. Barcelona, Paidós, 2001.)

${ }^{66}$ J. Rawls, A Theory of Justice, pp. 453-459. 
ción, es considerarlo sub specie aeternitatis; es tener en cuenta la situación humana no sólo desde todos los puntos de vista sociales, sino también desde todos los temporales". ${ }^{67}$ Su conclusión no puede ser más elocuente: "pureza de corazón, si uno pudiera alcanzarla, sería ver con claridad y actuar con la gracia del autocontrol desde este punto de vista". ${ }^{68}$ Su propuesta permanece como una referencia ejemplar ante el desastre. Después de largos años de enfermedad y padecimientos, que lo mantuvieron enclaustrado en Harvard, murió el miércoles 27 de noviembre del año 2002.

La resurrección de Kant en la propuesta y los argumentos de Rawls alecciona, pero poco puede hacer el espectro del hombre de Königsberg en este tiempo descompuesto, desajustado y que perdió sus goznes. ${ }^{69}$ Los hombres consideran legítimo vivir en ella, no porque en cada situación sean tratados como un fin y no un simple medio, ni porque reciban una trato igual los intereses de cada uno de ellos, sino porque la consideran perfectible. Lo político se pierde en la espiral violenta de la imposibilidad de la justicia.

\section{De la larga discusión académica a una mayor desigualdad}

Como se ve en las dos primeras secciones, durante la llamada Guerra Fría, en la que se enfrentaban un capitalismo en expansión y un socialismo lleno de problemas, con posiciones a favor y en contra en cuanto a sus matices éticos, la igualdad social fue un tema de primera línea en los círculos académicos. ${ }^{70}$ La opinión pública, sobre todo, la estadounidense, llamó "nuevos igualitarios" a los autores más destacados en la reelaboración de ese concepto. ${ }^{71}$ En esos mismos años, a través de protestas y organizaciones de ciudadanos, los desiguales de las sociedades capitalistas se movilizaban en torno a sus demandas. Estas avanzadas de la diversidad, además de reivindicar los principios liberales

${ }^{67}$ Ibid., p. 587.

${ }^{68}$ Idem.

${ }^{69}$ Vid. Philippe van Parijs, ¿Qué es una sociedad justa? Buenos Aires, Nueva Visión, 1992.

${ }^{70}$ Para los propósitos de este trabajo hay que agregar: Charles E. Lindblom y David K. Cohen, Usable Knowledge. New Haven/Londres, Universidad de Yale, 1979; Michael J. Sandel, Liberalism and the Limits of Justice. Nueva York, Universidad de Cambridge, 1982; Michael Walzer, Spheres of Justice A Defense of Pluralism and Equality. Nueva York, Basic Books, 1983; Jean Leca y Roberto Papini, dirs., Les démocraties sont-elles gouvernables? París, Economica, 1985; Charles R. Beitz, Political Equality An Essay in Democratic Theory. Princeton, N. J., Universidad de Princeton, 1989.

${ }^{71}$ Robert A. Nisbet, "Los costos de la igualdad", en Los humanistas y la política. México, FCE, 1984, p. 50. 
de igualdad ante la ley, así como el de oportunidades, luchaban por la "igualdad de resultados". 72

En el campo conservador reinaba el desconcierto y escaseaban los profetas. Popper era ante todo un filósofo de la física y sus incursiones en la teoría política se veían lejanas. The Open Society aparecía importante por sus apéndices y pocos aceptaban que Platón, Hegel y Marx fuesen representantes de sistemas cerrados y enemigos de la sociedad abierta, según se sostiene en ese libro. F. Hayek recibía el trato de perro muerto, no digamos de parte de los marxistas, quienes lo habían expulsado de la historia, sino hasta del pensamiento liberal. En su libro de 1971, Rawls no lo cita ni una sola vez, seguramente porque sólo lo consideraba un viejo premio Nobel de Economía, ya por entonces retirado en Suiza. Aún Douglas Rae, en 1981, apenas si lo menciona. ${ }^{73}$

Con la caída del Muro de Berlín, en 1989, y en la práctica, el fin del llamado "socialismo real", se despierta una euforia explicable en torno a la democracia liberal. ${ }^{74}$ No la comparten los más aguerridos defensores de la igualdad. ${ }^{75}$ Kennet Arrow, John Rawls y Amartya Sen, continúan produciendo nuevos argumentos para sus propuestas. ${ }^{76} \mathrm{El}$ debate se amplía y se vuelve cada vez más consistente, abarcando de manera decidida al Estado. ${ }^{77}$ Los estrellatos

${ }^{72}$ Ibid., p. 54 . En esa dirección aparecieron los libros de Christopher Jencks, Inequality (1972) y el de Herbert Gans, More Equality (1973). John Rawls con A Theory of Justice, en 1971, fue incluido por el propio Nisbet entre los "nuevos igualitarios" o los redentores socialistas.

${ }^{73}$ Douglas Rae, Equalities. Cambridge/Londres, Universidad de Harvard, 1981, p. 74, n. 6.

${ }^{74}$ Vid. Jacques Derrida, Espectros de Marx. Madrid, Trotta, 1995, p. 70. Véase también: Francis Fukuyama, The End of History and the Last Man. Nueva York, The Free Press, 1992, p. 27.

${ }^{75}$ Ver los artículos de Walzer y Elster en Emy Gutmann, ed., Democracy and the Welfare State. Princeton, N. J., Universidad de Princeton, 1988, pp. 13-26 y 53-78, respectivamente. Véase también: Nathan Glazer, The Limits of Social Policy. Cambridge, Mass., Universidad de Harvard, 1988; A. Sen, Inequality Reexamined. Nueva York, Clarendon, 1992, y A. Sen, On Ethics and Economics. Cambridge, Mass., Basil Blackwell, 1991.

${ }^{76}$ Vid. J. Rawls, "The Basic Liberties and Their Priority", en Sterling M. McMurrin, ed., Liberty, Equality and Law. Salt Lake City, Universidad de Utah, 1987, pp. 1-88; A. Sen, "Equality of What?", en S. M. McMurrin, ed., Liberty, Equality and Law, pp. 137-162.

${ }^{77}$ Vid. Robert Mangabeira U., Social Theory: Its Situation and Its Task. Nueva York, Universidad de Cambridge, 1987; M. Kaus, The End of Equality. Nueva York, Basic Books, 1992; S. Holmes, Passions and Constraint on the Theory of Liberal Democracy. Chicago/Londres, Universidad de Chicago, 1995; Michael J. Sandel, Liberalism and the Limits of Justice. Cambridge, Universidad de Cambridge, 1992; John Gray, Liberalism. Minneapolis, Universidad de Minnesota, 1986; David Held, Political Theory and the Modern State. Cambridge, G. B., Polity Press, 1984, y Milton Fisk, The State and Justice. Nueva York, Universidad de Cambridge, 1989. 
académicos se mantienen y aparecen nuevos, no sólo más jóvenes sino más arriesgados, más cercanos a los problemas políticos de la desigualdad. ${ }^{78}$

En la primera década del siglo XXI la desigualdad económica es más compleja y los modelos propuestos para superarla progresivamente aparecen inaplicables. Después de la caída del socialismo y ante el fracaso generalizado de los mecanismos del Estado de bienestar, subsidios, gasto público y empresas gubernamentales, la globalización económica se extiende sin horizonte alguno de referencia. Las cuestiones de justicia internacional y generacional aumentan su urgencia. ${ }^{79}$ Viejos problemas son repensados y se intentan marcar las coordenadas de los nuevos: hiperpotencia, agresividad de los nacionalismos, minorías étnicas y migración. ${ }^{80}$ El tema de la democracia se vuelve intempestivo, ¿qué democracia viene en la globalización? ${ }^{81}$

Cambian las preguntas. ¿La justicia se puede pensar en lo global? ¿Cómo aplicar una política económica global para la igualdad de oportunidades? ¿Qué esperar de la democracia en la globalidad? ¿Cuál podría ser hoy la gramática de la justicia? ${ }^{82}$ La globalización, de promesa se ha cambiado en amenaza. El círculo se ha roto. Sólo queda la complejidad, curvas hiperbólicas del adentro y el afuera. ${ }^{83}$ "La desigualdad es el más general de los hechos

${ }^{78}$ Vid. Michael Walzer, "The Communitarian Critique of Liberalism", en Political Theory, 18(1), 1990, pp. 6-23; M. Walzer, "The Idea of Civil Society. A Path to Social Reconstruction", en Dissent, primavera, 1991, pp. 293-304; M. Walzer, "Exclusion, Injustice, and The Democratic State", en Dissent, invierno, 1993, pp. 55-64; Th. Scanlon, "Promises and Practices", en Philosophy and Public Affairs, 19(3), 1990, pp. 199-226; Allen Buchanan, "Justice as Reciprocity versus Subject-Centered Justice", en Philosophy and Public Affairs, 19(3), 1990, pp. 227-252; Brian Barry, "Social Criticism and Political Philosophy", en Philosophy and Public Affairs, 19(4), 1990, pp. 360-373; A. Sen, "Justice: Means versus Freedoms", en Philosophy and Public Affairs, 19(2), 1990, pp. 111-121, y Adam Przeworski, Democracy and the Market. Cambridge, Universidad de Cambridge, 1991.

${ }^{79}$ Vid. Brian Barry, Theories of Justice. Berkeley, Universidad de California, 1989, parte II, cap. 5.

${ }^{80}$ Véase los artículos de Eric Hobsbaum, Alan Wolfe, Norman Birnbaum y Chantal Mouffe en M. Walzer, ed., Toward a Global Society. Providence/Oxford, Berghahn Books, 1995.

${ }^{81}$ Vid. Norberto Bobbio, "Democracy and the International System", en D. Archibugi y D. Held, eds., Cosmopolitan Democracy. Cambridge, M. A., Polity Press, 1995 , pp. 17-41; David Held, "Democracy and the International Order", en D. Archibugi y D. Held, eds., Cosmopolitan Democracy, pp. 96-120; Leo Panitch y Colin Leys, The End of Parliamentary Socialism From New Left to New Labour. Londres/Nueva York, Verso, 1997.

${ }^{82}$ Vid. Douglas Rae, Equalities. Cambridge/Londres, Universidad de Harvard, 1981, pp. 14,18 y 47.

${ }^{83}$ Vid. Peter Sloterdijk, En el mundo interior del capital. Madrid, Siruela, 2007, p. 26. 
sociales", pronóstico sin concesiones que Douglas Rae hizo con oportunidad sobre la situación. ${ }^{84}$

Paul Krugman, premio Nobel de Economía, al analizar el Informe Económico del Congreso de Estados Unidos, de 2013, trató el tema en su artículo semanal del New York Times. ${ }^{85}$ Sus afirmaciones son categóricas: "La desigualdad económica en Estados Unidos aumenta severamente". Se refiere, de inmediato, al dato más significativo de la situación. "Desde finales de los setenta, los salarios reales para la mitad de la fuerza de trabajo que gana menos, se han deteriorado o caído, mientras que el ingreso del uno por ciento que gana más casi se ha cuadruplicado (y los del 0.1 por ciento se ha incrementado todavía más)”. En México, la desigualdad empeora aún más. Si en Estados Unidos, el ingreso de la décima parte de las familias más pobres cabe 14 veces en el de las más ricas, en México esa proporción es 25 veces mayor.

Una desigualdad inexplicable, en particular, para los que la padecen. Krugman cita el refrán de Upton Sinclair: "Es difícil que alguien entienda algo cuando su salario depende de eso que no entiende". Pero, Krugman va más allá y se pregunta si habría llegado a su fin la vigencia del libro famoso y clásico de Bernard Mandeville, La fábula de las abejas o Los vicios privados hacen la prosperidad pública.

¿Cómo interpretar en el contexto del artículo este aumento de la desigualdad? De entrada se comprueba que la razón académica está cada vez más lejos de la razón pública y ésta de la razón de la democracia. La teoría de las decisiones racionales ha sido superada por las teorías financieras del capital virtual y por las estrategias de los derivados en la Bolsa. Nada se puede pensar fuera de esta virtualidad y de la democracia. Todos los reclamos han de dirigirse a estas instancias, aconseja Amartya Sen. ${ }^{86}$ Todos desembocan en la política y, por tanto, en la democracia, según James M. Buchanan. ${ }^{87} \mathrm{El}$ paso a dar sería en dirección de nuevas teorías de las decisiones públicas en contextos de alta complejidad. ${ }^{88}$

${ }^{84}$ Douglas Rae, "Maximin Justice and an Alternative Principle of General Advantage", en The American Political Science Review, 69(2), 1975, p. 630.

${ }^{85}$ P. Krugman, New York Times, 20 de enero de 2014, A15.

${ }^{86}$ A. Sen, La idea de la justicia, p. 424.

${ }^{87}$ James M. Buchanan, Constitutional Economics. Cambridge, Mass., Basil Blackwell, 1991, secciones 1, 2 y 3. Véase también J. M. Buchanan, Ensayos sobre economía política. México, Alianza, 1990.

${ }^{88}$ Vid. Niklas Luhmann, Political Theory in the Welfare State. Berlín/Nueva York, Walter de Gruyter, 1990; sobre todo cap. Ix: "The future of democracy". Véase también de Jon Elster: "Introduction", en Rational Choice. Nueva York, Universidad de Nueva York, 1986, pp. 1-33; El cemento de la sociedad. Barcelona, Gedisa, 1991; Una introducción a Karl Marx. México, Siglo XXI, 1991; Domar la suerte. Barcelona/Buenos Aires/México, Paidós, 1991; Justicia local. Barcelona, Gedisa, 1994; Ulises y las sirenas. 


\section{Perspectivas}

Un nuevo ciclo económico en su comienzo. Parece. Se caracteriza por los reacomodos de países y regiones en la globalización y por nuevos factores que inciden en ella de manera directa e inmediata. Durante estas semanas y meses y años, hiperreales e hipervirtuales, tres procesos no se dejan ver ni medir con exactitud.

Primero, aun si lento y vacilante, el de la recuperación de Estados Unidos al frente del nuevo rizo del capital. Segundo, el de los acuerdos para reactivar los movimientos regionales del mercado mundial, principalmente el Gran Tratado del Atlántico de Estados Unidos con la Unión Europea y el Acuerdo Transpacífico en el que, de nuevo, Estados Unidos aumentará su presencia en Asia, y en el que México, si eleva su competitividad, encontrará un motor adicional importante de su crecimiento durante los próximos años. Tercero, el desarrollo científico y tecnológico, no sólo en información y comunicación, sino también en las llamadas ciencias de la vida y en los conocimientos y dispositivos impensables que comienzan a diseñarse a partir de la nueva toma de energía en el universo: computadoras cuánticas, técnicas alternativas de recarga de energía y virtualización de las distintas formas de producir cosas.

Nuevas composiciones de fuerzas atraviesan la vida. Han comenzado a aparecer otros lenguajes. Inician otros modos de interacción en el vivir-juntos caracterizados por su dimensión experimental. Se vislumbra, ya, otra civilización. Se caracteriza por su virtualidad y su velocidad, aunque avanza en medio de la violencia, la dureza, y las desviaciones. Aumenta la aleatoriedad: todo es metaprobabilístico, todo se juega en una superlotería.

Frente a esta avalancha civilizatoria, las viejas preguntas caen por su propio peso, son obsoletas. Naturalmente, las cuestiones concretas deberán resolverse de acuerdo a la máxima intensidad estratégica, pero pronto se convertirían en curiosidades de museo si no vislumbran, también aquí, tres giros. Tienen que ver con el hacer lugar. Primero, generación de empleos. Segundo: transformación de los entornos para que favorezcan la velocidad individual. Tercero: giro de la creatividad. Esto exige enlaces y decisiones sistémicos. La economía no puede separarse de la educación, y ambas de lo que se llama cultura: modos de pensar y de ser.

Se trata de imprimir flexibilidad y una polirracionalidad estratégica a la economía. Esto significa introducir en ella axiomas no económicos: éticos y políticos, por ejemplo. El singular de las libertades, al participar en el juego del mercado, no se encuentra en una situación de libertad sino en medio de una

México, FCE, 1995; Psicología política. Barcelona, Gedisa, 1995, y Ulises desatado. Barcelona. Gedisa, 2002. 
lógica conductista insuperable. Es cuestión de flexibilizar este conductismo, lo que demanda, a su vez, la flexibilización de la estructura social.

La gran tarea de una posible "filosofía de la economía" sería la de dar nueva vida a una vieja idea: la economía es una ciencia política y moral. ${ }^{89}$ Esto exige salir de la filosofía e irrumpir en la política. La democracia está a la orden del día.

En esta dirección se encontrarían las diversas interpretaciones que Amartya Sen ofrece del teorema de la imposibilidad de Arrow. Al transformarlo en teorema de las posibilidades o en teorema de la indiferencia, se acerca a las propuestas de Buchanan de la reconstrucción de la economía a partir de un axioma distinto al de la maximización de la ganancia individual. Se trataría, entonces, de hacer llegar a la economía una racionalidad forjada socialmente. Esta racionalidad pasa por el crisol de la democracia por venir.

${ }^{89}$ Vid. Bernard Stiegler, Pour une nouvelle critique de l'économie politique. París, Galilée, 2009. 